\title{
Protumorigenic M2-like phenotype cell infiltration in the (1) melanotic neuroectodermal tumor of infancy $\underline{s}$
}

Luciana Strieder, DDS, ${ }^{a}$ Román Carlos, DDS, ${ }^{\mathrm{b}}$ Jorge Esquiche León, DDS, PhD, ${ }^{\mathrm{c}}$ Alfredo Ribeiro-Silva, MD, PhD, ${ }^{\mathrm{d}}$ Victor Costa, DDS, ${ }^{\mathrm{a}}$ and Estela Kaminagakura, DDS, $\mathrm{PhD}^{\mathrm{e}}$

Institute of Science and Technology, UNESP-Univ Estadual Paulista, São José dos Campos, Brazil; University of São Paulo, Ribeirão Preto, Brazil; and Centro Clínico de Cabeza y Cuello, Guatemala City, Guatemala.

Objective. The aim of this study is to report 2 cases of melanotic neuroectodermal tumor of infancy (MNTI), emphasizing the analysis of intratumoral immune cells by immunohistochemistry.

Study Design. Case 1: A 6-month-old girl presented with a 3-cm tumor in the anterior region of the left maxilla. Case 2: A 4-month-old boy presented with a 4-cm tumor in the anterior region of the left maxilla. Microscopically, case 1 had predominantly neuroblast-like cells supported by fibrillary neuropil-like stroma arranged in an alveolar pattern, whereas case 2 exhibited scattered melanocyte-like and neuroblast-like cells supported by fibrovascular stroma. A large immunohistochemical panel for characterizing intratumoral macrophage and dendritic cell subsets was performed. Results. Immunohistochemical analysis indicated positivity for HLA-DR, XIIla, CD68, and CD163 (range 6\%-50\%) mainly on the fibrovascular stroma, suggesting M2 macrophage-like cell phenotype. CD138 was overexpressed in the tumor stroma. Conclusions. Results suggest the involvement of M2-polarized macrophages in the MNTI pathogenesis, which may act by modulating tumor growth and/or tumor stromal remodeling. (Oral Surg Oral Med Oral Pathol Oral Radiol 2016;121:173-179)

Melanotic neuroectodermal tumor of infancy (MNTI) is a rare neoplasia that often occurs in children younger than 1 year old. ${ }^{1,2}$ The anterior maxilla is affected in about $60 \%$ of cases, ${ }^{3}$ but there are also reports about other parts of the body. 4

Microscopically, MNTI is characterized by being biphasic, containing epithelioid cells (melanocyte-like), ${ }^{1}$ broad and polygonal with variable deposits of melanin. ${ }^{3,4}$ The other component is composed of small, round blue cells (neuroblast-like) located in the center of the nests. ${ }^{1}$ In addition, neurofibrillary material resembling glial tissue and variable deposition of connective tissue stroma can be visualized. ${ }^{2,3}$

Although most studies evaluate the immunohistochemical (IHC) profile of MNTI tumor cells, there are no data assessing stromal cells and/or intratumoral immune cells in MNTI. Thus, by IHC analysis, the larger cells

This work was presented as poster in XXIII Brazilian Congress of Stomatology and Oral Pathology in July, 2015.

${ }^{\mathrm{a}}$ Master Student of Oral Biopathology, Institute of Science and Technology, UNESP-Univ Estadual Paulista, School of Dentistry, São José dos Campos, Brazil.

${ }^{\mathrm{b}}$ Director of Centro Clínico de Cabeza y Cuello, Guatemala City, Guatemala.

${ }^{c}$ Professor of Oral Pathology, Department of Stomatology, Public Oral Health and Forensic Dentistry, University of São Paulo, Ribeirão Preto School of Dentistry, Ribeirão Preto, Brazil.

${ }^{\mathrm{d}}$ Department of Pathology, Ribeirão Preto Medical School, University of São Paulo, Ribeirão Preto, Brazil.

${ }^{\mathrm{e}}$ Professor of Stomatology, Department of Bioscience and Oral Diagnosis, Institute of Science and Technology, UNESP-Univ Estadual Paulista, School of Dentistry, São José dos Campos, Brazil. Received for publication Jun 12, 2015; returned for revision Aug 31, 2015; accepted for publication Sep 20, 2015.

(c) 2016 Elsevier Inc. All rights reserved.

$2212-4403 / \$$ - see front matter

http://dx.doi.org/10.1016/j.00oo.2015.09.015 express cytokeratins (CKs), epithelial membrane antigen (EMA), glial fibrillary acidic protein (GFAP), S100 protein, and HMB45, and the smaller cells express CD56 and synaptophysin; both cells express neuron-specific enolase (NSE), PGP 9.5, and chromogranin A. ${ }^{4,5}$

Several studies have found that the tumor microenvironment plays an important role in neoplasm development and behavior. ${ }^{6}$ The tumor microenvironment consists of stromal cells, blood vessels, and immune cell infiltrates. Intratumoral immune cells may play a dual role in tumor development and progression. Thus, infiltrating immune cells can either eliminate tumor cells or recruit other immune cells to favor tumor growth and progression. ${ }^{6}$ Within this group of intratumoral immune cells, macrophages and dendritic cells (DCs) play a pivotal role that is known to affect progression in many human malignancies. Infiltration by mature, active DCs and/or M1-polarized macrophages elicits immune activation and recruitment of immune effector cells, whereas infiltration by immature DCs and/or M2-polarized macrophages imparts immune-suppressive properties or a protumorigenic profile. $^{7}$

\section{Statement of Clinical Relevance}

This is the first report that emphasizes the importance of the interaction of tumor cells and microenvironment in the pathogenesis of melanotic neuroectodermal tumor of infancy. Understanding the biological mechanisms of tumor development can support therapeutic decision-making in pediatric patients. 

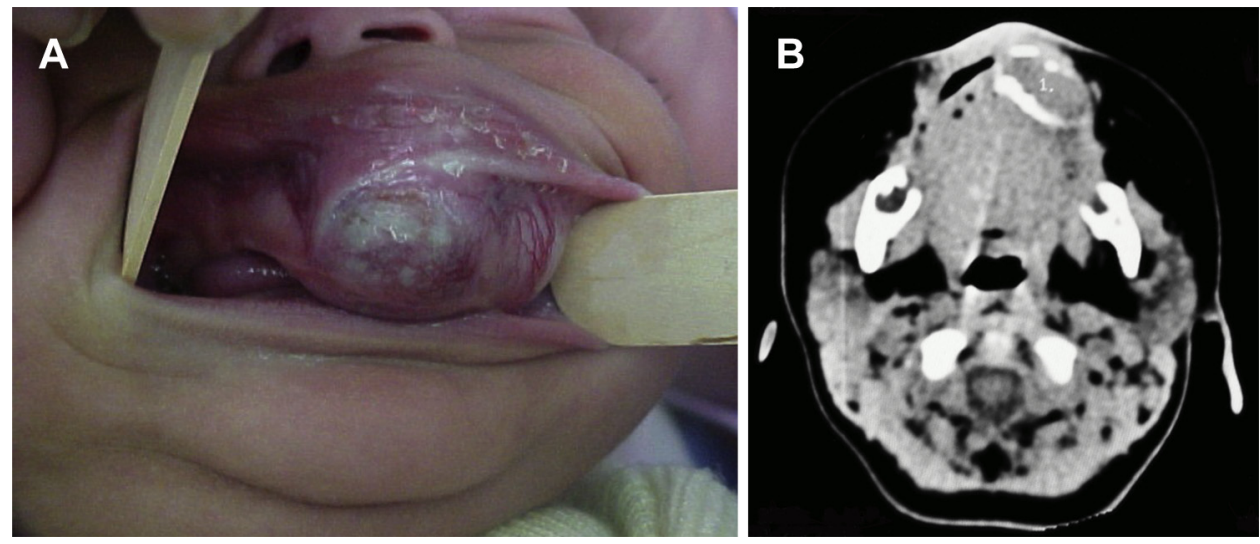

Fig. 1. Case 1. (A) Initial clinical appearance showing a destructive mass of the anterior region of the maxilla. (B) Well-defined hypodense area with cortical disruption was observed on computed tomographic scanning.

In the present study we have analyzed, characterizing macrophage and DC subsets by IHC, the immune cell infiltration in 2 MNTI cases and discuss its role in the pathogenesis of this type of tumor.

\section{CASE REPORTS}

\section{Case 1}

A 6-month-old girl was referred to the Cabeza y Cuello Clinical Diagnosis Service of Guatemala City with a 3-cm brown-grayish osteolytic lesion in the anterior region of the left maxilla, causing facial asymmetry and disruption of cortical bone, with 5 months of evolution. During the interview, the mother reported difficulties in breastfeeding. Computed tomography was performed and revealed a hypodense mass located in the anterior maxilla of $3.5 \mathrm{~cm}$, with disruption of the vestibular cortical bone (Figure 1A, B). With a clinical diagnosis of MNTI, the patient underwent in-hospital surgical excision. Microscopically, neoplastic proliferation was observed containing groups of primitive neuroectodermal cells, intensely hyperchromatic, delimited by septa of moderately vascular fibrocollagenous tissue. Neuroblast-like cells supported by glial-like tissue in association with sparse melanocyte-like cells were observed. Some of the melanocyte-like cells contained abundant melanin pigment. The definitive diagnosis of MNTI was established (Figure 2A, B). The patient has shown no signs of disease recurrence in 72 months of follow-up.

\section{Case 2}

A 4-month-old boy was referred to the Cabeza y Cuello Clinical Diagnosis Service of Guatemala City with an osteolytic lesion in the anterior region of the left maxilla of 4-cm in diameter, with facial asymmetry, disruption of cortical bone, and 4 months of evolution (Figure 3). The mother reported difficulties in breastfeeding. With a clinical diagnosis of MNTI, the patient underwent inhospital surgical excision. Microscopic examination found a biphasic population, made up of nests of hyperchromatic cells, supported by a large and dense fibrocollagenous stroma. The cell nests were composed of cuboidal cells of epithelioid aspect, as well as of small cells. These epithelioid cells (melanocyte-like cells) exhibited vesicular nuclei and cytoplasm with melanin granules. The second cell type of the nests consisted of neuroblast-like cells, with small, round, and hyperchromatic nuclei, surrounded by scant cytoplasm. Mitotic activity was not observed. The definitive diagnosis of MNTI was established (Figure 4A, B). The patient has shown no signs of disease recurrence in 5 years of follow-up.

\section{Immunohistochemical reaction}

All tissue specimens were fixed in $10 \%$ neutral-buffered formalin for 24 hours at room temperature, embedded in paraffin at $55^{\circ} \mathrm{C}$, and cut into consecutive parallel $3-\mu \mathrm{m}$ thick sections. For immunohistochemistry, the slides were hydrated and treated with hydrogen peroxide.

For retrieval of the CD56, CD57, S100, XIIIa, CD1a, CD207, CD138, CD68, CD163, HLA-DR, CD11c, CD209, CD123, CD303, CD80, CD83, and Ki-67 epitopes (Table I), the tissue specimens were pretreated with $10 \mathrm{mM}$ sodium citrate buffer, $\mathrm{pH} 6.0$, in a pressure cooker. The sections were then successively incubated with the primary antibodies. Next, the sections were incubated with the secondary antibodies conjugated with streptavidin-biotinperoxidase (K0690; Universal Dako LSAB ${ }^{\circledR}+$ Kit, Peroxidase, Dako, Carpinteria, CA, USA). The reactions were developed with diaminobenzidine and the sections were counterstained with Carazzi's hematoxylin. In case of doubts, the hematoxylin-eosin slide was used for comparison; the melanin pigmentation was restricted to melanocyte-like cells. A red chromogen was used if doubts persisted in the immunostaining analysis. Moreover, these cells are well characterized by immunohistochemistry. ${ }^{5}$ The percentage of positive cells was calculated ${ }^{8}$ with the aid of an image computer analyzer (Dako Automated Cellular Imaging System; Dako, Carpinteria, CA, USA) in a blinded analysis performed independently by the authors (J.E.L. and L.S.). It was classified as negative $(0 \%-5 \%$ of positivity), $+(6 \%-50 \%)$ or $++(>50 \%)$ 

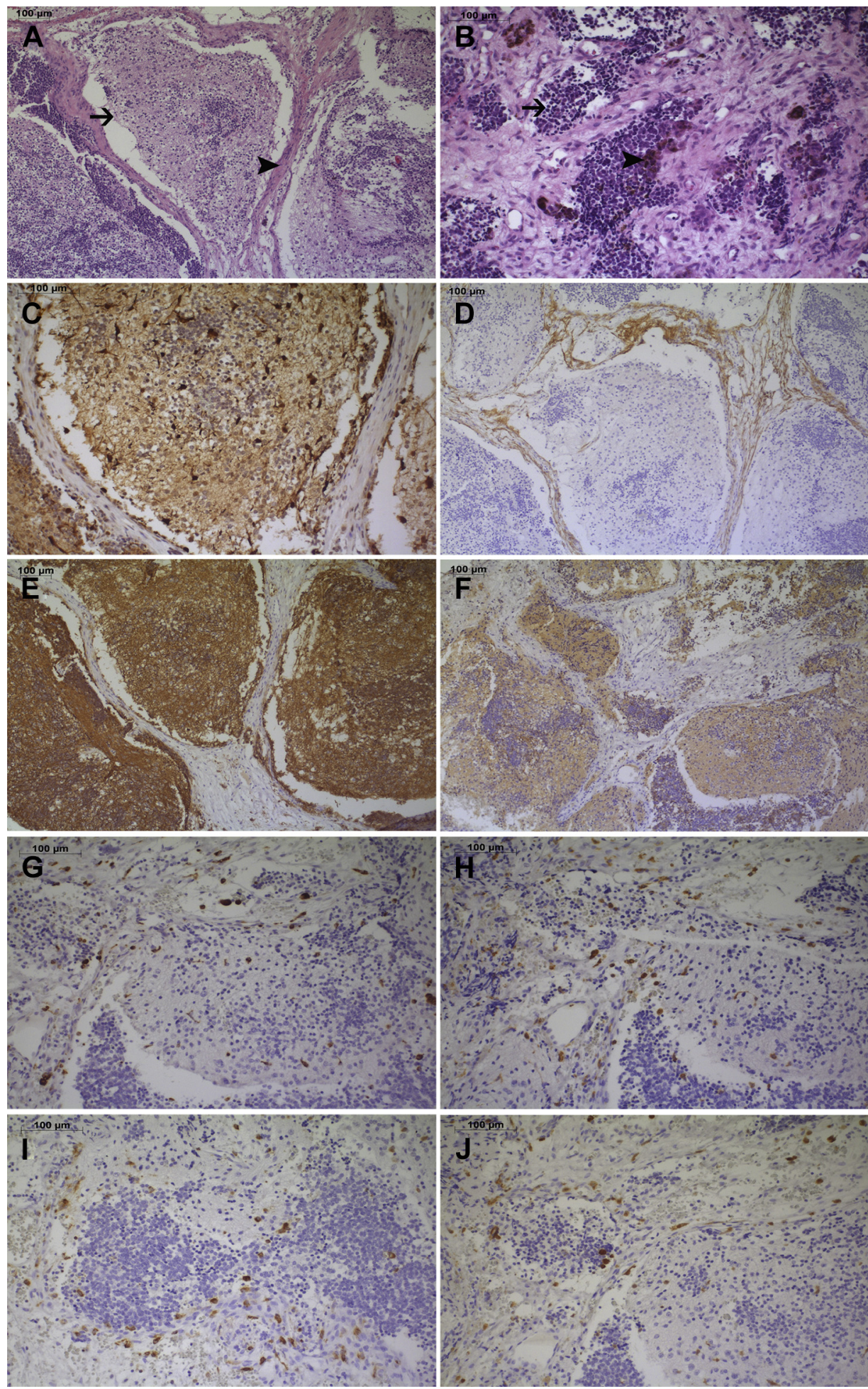

Fig. 2. Case 1. (A) Photomicrographs showing glial-like cells (arrow) and connective tissue septa (arrowhead). (B) Proliferation of neuroblast-like cells (arrow) and focal area containing pigmented melanocyte-like cells (arrowhead). (C) S100 protein was positive on melanocyte-like cells, dendritic-like cells, and glial-like tissue, whereas (D) CD138 highlighted the stroma cells and (E) gliallike tissue and neuroblast-like cells were strongly positive for CD56 and (F) weakly positive for CD57. Consecutive tissue sections showed immunoexpression for (G) CD68, (H) CD163, (I) HLA-DR, and (J) XIIIa. (A and B, hematoxylin-eosin stain; C-J, immunohistochemistry.) A high-resolution version of this slides for use with the Virtual Microscope is available as eSlide: CD11c VM01106; CD138 VM01108; CD163 VM01111; CD56 VM01109; CD57 VM01107; CD68 VM01110; H\&E VM01112; HLA-DR VM01115; Ki67 VM01118; S100 VM01119; XIIIa VM01113. 


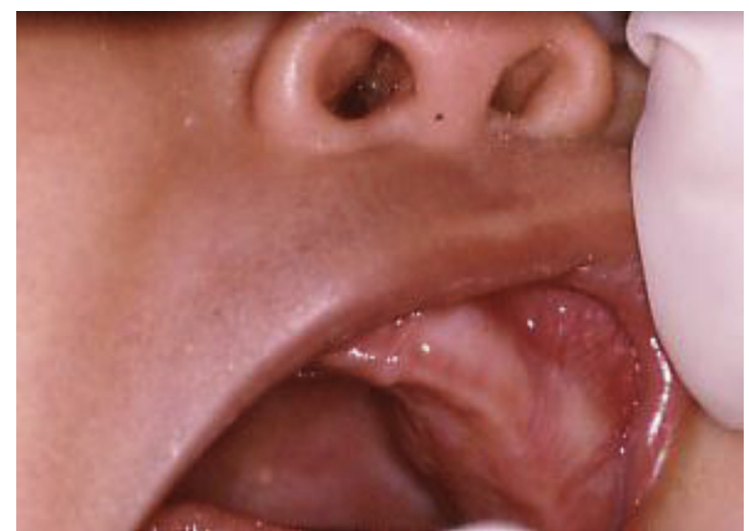

Fig. 3. Case 2: Intraoral view with enlargement of the anterior maxilla region.

\section{Immunohistochemical findings}

The tumor cells and glial-like tissue were positive for CD56 and CD57, the former presenting strong staining. The melanocytelike cells were $\mathrm{S} 100$ positive in both cases, whereas only case 1 exhibited isolated S100-positive dendritic-like cells on the glial-like tissue.

Dendritic-like cells positive for HLA-DR, XIIIa, CD68, and CD163 (6\%-50\%) were observed in the fibrous septa and gliallike tissue of case 1 and in the tumor stroma of case 2 . These dendritic-like cells in the glial-like tissue and in tumor stroma appeared to be microglia-like cells and macrophage-like cells, respectively.

Interestingly, we observed positivity for CD138 in the tumor stroma in both cases, being slightly more intense in case 1 (Table I). Immunomarkers for DC subtypes, such as Langerhans-like cells (CD1a and CD207), myeloid DCs (myDCs) (CD11c), mature DCs (CD80 and CD83), submucosal DCs (CD209), as well as plasmacytoid DCs (pDCs) (CD123 and CD303), were all negative (Table I; Figures 2 and 4C-J). In contrast to case 2 (proliferative index, $1 \%$ ), case 1 , which had large areas containing tumor cells and scarce tumor stroma, exhibited a higher proliferative index (10\%).

\section{DISCUSSION}

MNTI presents itself with 2 populations of cells; 1 contains sheets of large epithelioid cells, ${ }^{1}$ with clear and abundant cytoplasm with intracellular granular melanin pigmentation $^{2}$; the other consisting of small round cells with hyperchromatic nuclei and scant cytoplasm. ${ }^{2}$ These neuroblast-like cells are considered the aggressive component of the lesion. ${ }^{1}$ In this study, case 1 had a higher expression of $\mathrm{Ki}-67$ in the neuroblast-like cells and CD138 in the stromal cells compared with case 2. CD138 expression by stromal fibroblasts may be associated with proliferation of neoplastic cells through activation of the signaling pathway via fibroblast growth factor 2 (FGF2) and stromal cell-derived growth factor-1 (SDF1). ${ }^{9}$ Thus, the concept of tumor-stromal cell interactions may be considered in these 2 MNTI cases. Indeed, it has been found that CD138 modulates the adherence of malignant neoplastic cells to the extracellular matrix, preventing motility, cell invasion, and consequently metastasis in the malignant neoplasms. ${ }^{10,11}$

On the other hand, it has been found that the interaction between tumor cells and immune cells is much more dynamic and complex than been previously thought. The tumor microenvironment plays an important role in the development and behavior of neoplasms. ${ }^{12}$ Several studies emphasizing immune cell to tumor cell interactions have reported that infiltrating immune cells are composed primarily of TCD4+ and TCD8+ cells, regulatory $\mathrm{T}$ cells (Tregs), DCs, and macrophages. ${ }^{7}$ Immune cells have an inherent plasticity when responding to suppressive or stimulatory cytokines. ${ }^{13}$ Depending on the tumor stage, immune cells may display different properties, either a pro- or an antitumoral profile, highlighting the importance of studying the antitumor response in the course of the tumor development. ${ }^{14,15}$ In the initial phase of carcinogenesis, immune cells play an antitumor role, having the ability to destroy tumoral cells; however, they may also undergo phenotypic changes and favor growth and tumor progression. ${ }^{13-15}$

M1-polarized macrophages play an important role in the T-helper (Th) 1 response and are effective in the response to tumor cells. ${ }^{12}$ In the initial phase of tumorigenesis, Ly-6 $\mathrm{C}^{\text {high }}$ monocytes/DCs exhibit strong antitumor properties, in part through a reactive oxygen species (ROS)-dependent mechanism. ${ }^{15}$ Several chemical mediators, particularly interleukin (IL)-10, favor the conversion of M1 macrophages (with antitumor properties) to the protumoral M2 phenotype, because it inhibits monocyte/DC recruitment and/or differentiation. ${ }^{13,15,16}$ Macrophages associated to tumors express Ly6 a and CD81, which play a role in the interaction of antigen-presenting cells (APCs) with T cells, and in the maintenance of the Th2 phenotype. Therefore, these molecules may contribute to changes in the adaptive immune response at the tumor site in a Th2 direction. ${ }^{17}$ In this study, it was shown, that MNTI can exhibit a population of intratumoral immune cells. Indeed, it was observed that cells usually exhibiting a dendritic morphology and located in the glial-like tissue and tumor stroma are positive for HLA-DR, XIIIa, CD68 and CD163, suggesting the presence of M2polarized macrophages-like cells. Because of its protumor phenotype, it is suggested that these cells contribute to the survival of tumor cells and the progression of MNTI, as reported by several authors. ${ }^{17,18}$ Tumor cells produce various molecules that interact with the neighboring cells, modulating their functions to the tumor cells' advantage. ${ }^{18}$ An example of this is when tumor cells from breast and skin overexpress a2 

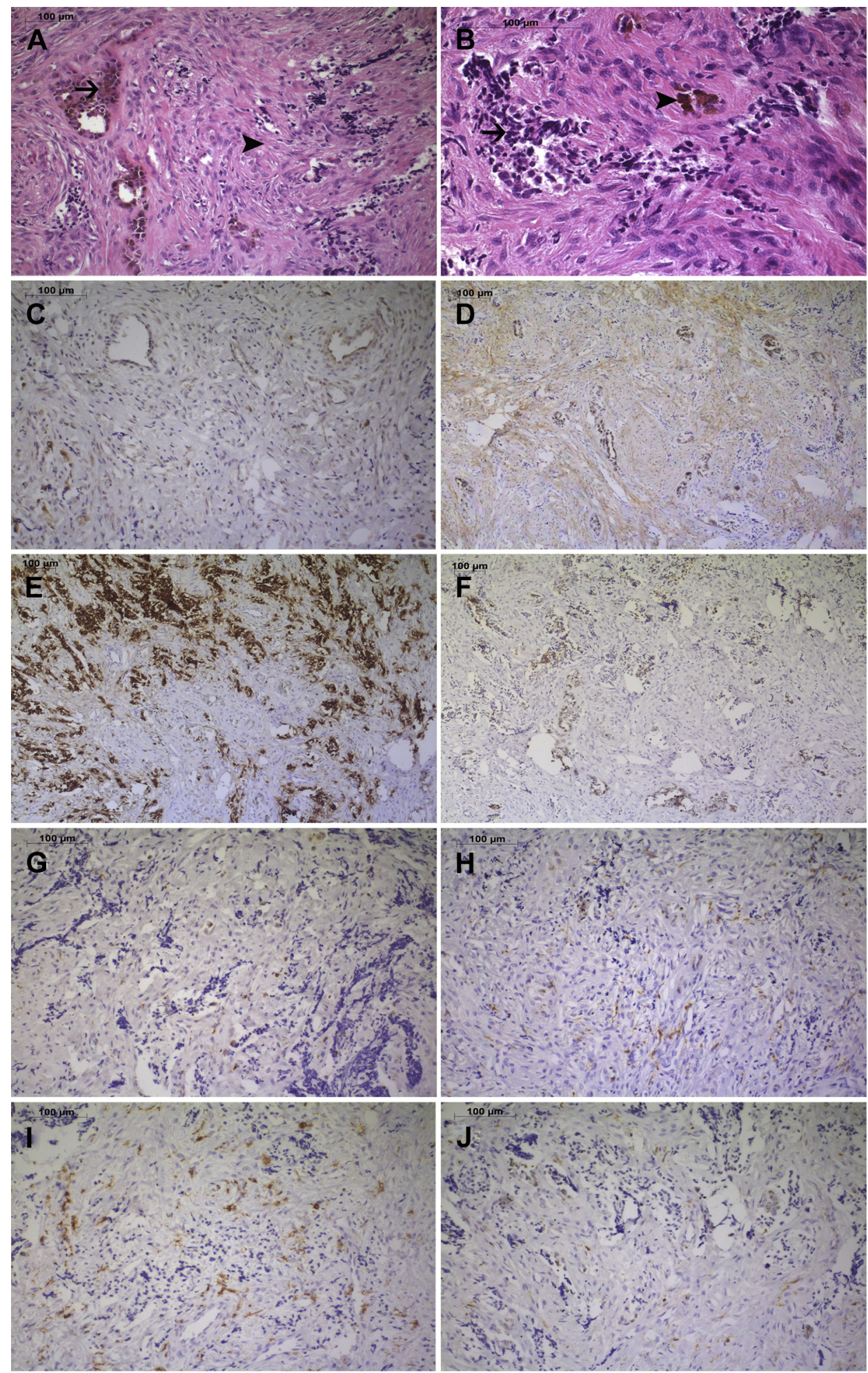

Fig. 4. Case 2. (A) Focal proliferation of pigmented melanocyte-like cells (arrow) and fibrous stroma (arrowhead). (B) In detail, pigmented melanocyte-like cells (arrowhead) and neuroblast-like cells (arrow). (C) S100 protein was positive on melanocyte-like cells, and (D) CD138 was positive on stroma cells. (E) CD56 was strongly positive on melanocyte-like and neuroblast-like cells, whereas (F) CD57 was weakly positive. Consecutive tissue sections showed for (G) CD68, (H) CD163, (I) HLA-DR, and (J) XIIIa. (A and B, hematoxylin-eosin stain; C-J, immunohistochemistry.) A high-resolution version of this slides for use with the Virtual Microscope is available as eSlide: CD11c VM01120; CD138 VM01130; CD163 VM01138; CD56 VM01129; CD57 VM01122; CD68 VM01126; H\&E VM01142; HLA-DR VM01144; Ki67 VM01140; S100 VM01123; XIIIa VM01146. 
Table I. List of primary antibodies and results for both cases

\begin{tabular}{|c|c|c|c|c|c|c|c|c|c|c|}
\hline \multirow[b]{2}{*}{ Antibody } & \multicolumn{4}{|c|}{ Case 1} & \multicolumn{3}{|c|}{ Case 2} & \multirow[b]{2}{*}{ Dilutions } & \multirow[b]{2}{*}{ Clone } & \multirow[b]{2}{*}{ Supplier } \\
\hline & $M$ & $N$ & $G$ & $S$ & $M$ & $N$ & $S$ & & & \\
\hline$\overline{\mathrm{CD} 56}$ & ++ & ++ & ++ & - & + & ++ & - & $1: 100$ & CD564 & Leica Biosystems \\
\hline CD57 & + & ++ & ++ & - & + & + & - & $1: 500$ & TB01 & DakoCytomation $^{\dagger}$ \\
\hline S100 & + & - & + & - & + & - & - & $1: 300$ & Polyclonal & Leica Biosystems \\
\hline XIIIIa & - & - & + & + & - & - & + & $1: 100$ & E980.1 & Leica Biosystems \\
\hline CD1a & - & - & - & - & - & - & - & $1: 400$ & 010 & DakoCytomation $^{\dagger}$ \\
\hline CD207 & - & - & - & - & - & - & - & $1: 200$ & 12 D6 & Monosan \\
\hline CD138 & - & - & - & ++ & - & - & + & $1: 500$ & MI15 & DakoCytomation $^{\dagger}$ \\
\hline CD68 & - & - & + & + & - & - & + & $1: 3000$ & $\mathrm{KP} 1$ & DakoCytomation ${ }^{\dagger}$ \\
\hline CD163 & - & - & + & + & - & - & + & $1: 500$ & 10 D6 & Leica Biosystems* \\
\hline HLA-DR & - & - & ++ & ++ & - & - & ++ & $1: 500$ & TAL.1 B5 & DakoCytomation $^{\dagger}$ \\
\hline CD11c & - & - & - & - & - & - & - & $1: 500$ & $5 \mathrm{D} 11$ & Leica Biosystems \\
\hline CD209 & - & - & - & - & - & - & - & $1: 2000$ & Polyclonal & $\mathrm{Abcam}^{\S}$ \\
\hline CD123 & - & - & - & - & - & - & - & $1: 200$ & BR4 MS & Leica Biosystems \\
\hline CD303 & - & - & - & - & - & - & - & $1: 500$ & $124 \mathrm{~B} 3.13$ & Dendritics \\
\hline CD80 & - & - & - & - & - & - & - & $1: 100$ & Polyclonal & Abcam $^{\S}$ \\
\hline CD83 & - & - & - & - & - & - & - & $1: 100$ & $1 \mathrm{H} 4 \mathrm{~b}$ & Leica Biosystems* \\
\hline $\mathrm{Ki}-67$ & $>10 \%$ & & & & $>1 \%$ & & & $1: 500$ & MIB-1 & DakoCytomation $^{\dagger}$ \\
\hline
\end{tabular}

$M$, melanocyte-like cells; $N$, neuroblast-like cells; $G$, glial-like cells; $S$, stroma.

$-(0 \%-5 \%$ of positivity), $+(6 \%-50 \%)$, or $++(>50 \%)$.

*Newcastle, UK.

${ }^{\dagger}$ Glostrup, Denmark.

${ }^{\ddagger}$ Uden, Netherlands.

${ }^{\S}$ Cambridge, UK.

isoform of the "a" subunit of V-ATPase $(\mathrm{a} 2 \mathrm{~V})$ on their surface to modulate the phenotypic conversion of resident macrophages (a2M $\Phi$ ) mainly into the M2polarized type, through the action of $\mathrm{N}$-terminal domain of a2 $\mathrm{V}$ (a2NTD). These macrophages produce multiple factors, such as mannose receptor 1 , arginase 1 , IL-10, and transforming growth factor $\beta$, matrix metalloproteinase 9, and vascular endothelial growth factor, that contribute to angiogenesis, invasion, survival, and progression of the tumor. In addition, a2 M $\Phi$ cocultured with naive $\mathrm{T}$ cells inhibited $\mathrm{T}$-cell activation. ${ }^{18}$

Macrophages and DCs represent variations of maturation/activation stages of mononuclear phagocytes. DCs are potent APCs with the ability to prime naïve T cells, and play an important role in the initiation and regulation of immune responses. ${ }^{14,19,20}$ The DC lineage is not well characterized in human neoplasms ${ }^{7}$; however, 2 main populations of DCs, pDCs and myDCs, have been recognized. ${ }^{7,21}$ Patients carrying mutations in $I R F 8^{22}$ and $G_{A T A 2^{23}}$ lack all blood DC subsets, which suggests a common origin. $^{21}$ However, patients affected with a mutation in GATA2 had a normal number of epidermal Langerhans cells, indicating a distinct origin from pDCs and myDCs. ${ }^{23}$ Human DC phenotypes show a functional specialization regarding pathogen recognition, cytokine secretion, CD8+ cytotoxic, and $\mathrm{CD} 4+\mathrm{T}$ cell responses ${ }^{21}$ and tumor neovascularization. ${ }^{19}$ This characterizes the plasticity of these cells. Tumor-infiltrating DCs (TIDCs) have a controversial role on the prognosis of different types of cancers. ${ }^{7}$ In melanoma, TIDCs are correlated to regression, ${ }^{24}$ whereas in a murine model of ovarian cancer, depleting DCs early in the course of the disease accelerates tumor progression. However, in a more advanced stage, they suppress tumor progression. ${ }^{14}$ Tumoral stroma provides chemokines, blood supply, and extracellular matrix and removes waste and dead cells. This dynamic process can have a negative effect on DC development and function. ${ }^{19}$ The immature TIDCs are found within the tumor, whereas the mature TIDCs are localized in the peritumoral area. ${ }^{25}$ The STAT family are responsible for DC maturation. ${ }^{19}$ In the present study, numerous cells with dendritic-appearing morphology were observed, almost all negative for CD80 and CD83. These findings lead to consider an immature TIDC phenotype. However, the comprehensive analysis of the used immunomarkers suggests that the intratumoral immune cells identified in these 2 MNTI cases belong to a continuous morphologic and functional spectrum between DCs and macrophages. Both MNTIs have nearly similar proportions of M2-like phenotype cells; thus, it is suggested that they participate in tumorigenesis. Other studies are needed to validate our findings and determine, if possible, the prognostic and/ or therapeutic implications.

Luciana Strieder (Decision 028/2014) and Victor Costa (Decision 070/2014) are recipient of a master's fellowship 
from Fundação de Amparo à Pesquisa do Estado do Amazonas (FAPEAM).

\section{REFERENCES}

1. Gaiger de Oliveira M, Thompson LD, Chaves AC, Rados PV, da Silva Lauxen I, Filho MS. Management of melanotic neuroectodermal tumor of infancy. Ann Diagn Pathol. 2004;8: 207-212.

2. Selim H, Shaheen S, Barakat K, Selim AA. Melanotic neuroectodermal tumor of infancy: review of literature and case report. J Pediatr Surg. 2008;43:E25-29.

3. Pattanayak Mohanty S, Ray JG, Richa, Mukherjee S, Mandal C, Chaudhuri K. Melanotic neuroectodermal tumour of infancy. BMJ Case Rep. 2010;2010.

4. Bellarbi S, Harmouch A, El Ochi MR, Fikri M, Arkha Y, Sefiani S. Melanotic progonoma of temporal and occipital bones: a case report. Neurochirurgie. 2013;59:138-140.

5. Barrett AW, Morgan M, Ramsay AD, Farthing PM, Newman L, Speight PM. A clinicopathologic and immunohistochemical analysis of melanotic neuroectodermal tumor of infancy. Oral Surg Oral Med Oral Pathol Oral Radiol Endod. 2002;93:688698.

6. Galdiero MR, Bonavita E, Barajon I, Garlanda C, Mantovani A, Jaillon S. Tumor associated macrophages and neutrophils in cancer. Immunobiology. 2013;218:1402-1410.

7. Tran Janco JM, Lamichhane P, Karyampudi L, Knutson KL. Tumor-infiltrating dendritic cells in cancer pathogenesis. J Immunol. 2015;194:2985-2991.

8. Bocchi EA, Tanigawa RY, Brandão SM, et al. Immunohistochemical quantification of inflammatory cells in endomyocardial biopsy fragments after heart transplantation: a new potential method to improve the diagnosis of rejection after heart transplantation. Transplant Proc. 2014;46:1489-1496.

9. Su G, Blaine SA, Qiao D, Friedl A. Shedding of syndecan-1 by stromal fibroblasts stimulates human breast cancer cell proliferation via FGF2 activation. J Biol Chem. 2007;282:14906-14915.

10. Teng YH, Aquino RS, Park PW. Molecular functions of syndecan-1 in disease. Matrix Biol. 2012;31:3-16.

11. Anttonen A, Kajanti M, Heikkilä P, Jalkanen M, Joensuu H. Syndecan-1 expression has prognostic significance in head and neck carcinoma. Br J Cancer. 1999;79:558-564.

12. Biswas SK, Allavena P, Mantovani A. Tumor-associated macrophages: Functional diversity, clinical significance, and open questions. Semin Immunopathol. 2013;35:585-600.

13. Smith HA, Kang Y. The metastasis-promoting roles of tumorassociated immune cells. J Mol Med (Berl). 2013;91:411-429.

14. Scarlett UK, Rutkowski MR, Rauwerdink AM, et al. Ovarian cancer progression is controlled by phenotypic changes in dendritic cells. J Exp Med. 2012;209:495-506.
15. Pommier A, Audemard A, Durand A, et al. Inflammatory monocytes are potent antitumor effectors controlled by regulatory CD4+ T cells. Proc Natl Acad Sci U S A. 2013;110:1308513090.

16. Sica A, Schioppa T, Mantovani A, Allavena P. Tumour-associated macrophages are a distinct M2 polarised population promoting tumour progression: potential targets of anti-cancer therapy. Eur J Cancer. 2006;42:717-727.

17. Biswas SK, Mantovani A. Macrophage plasticity and interaction with lymphocyte subsets: cancer as a paradigm. Nat Immunol. 2010;11:889-896.

18. Katara GK, Jaiswal MK, Kulshrestha A, Kolli B, GilmanSachs A, Beaman KD. Tumor-associated vacuolar ATPase subunit promotes tumorigenic characteristics in macrophages. Oncogene. 2014;33:5649-5654.

19. Fricke I, Gabrilovich DI. Dendritic cells and tumor microenvironment: a dangerous liaison. Immunol Invest. 2006;35: 459-483.

20. Chen J, Yang J, Jiang J, Zhuang Y, He W. Function and subsets of dendritic cells and natural killer cells were decreased in gastric cancer. Int J Clin Exp Pathol. 2014;7:8304-8311.

21. Durand M, Segura E. The known unknowns of the human dendritic cell network. Front Immunol. 2015;6:129.

22. Hambleton S, Salem S, Bustamante J, et al. IRF8 mutations and human dendritic-cell immunodeficiency. $N$ Engl J Med. 2011;365:127-138.

23. Bigley V, Haniffa M, Doulatov $\mathrm{S}$, et al. The human syndrome of dendritic cell, monocyte, B and NK lymphoid deficiency. $J$ Exp Med. 2011;208:227-234.

24. Ladányi A, Kiss J, Somlai B, et al. Density of DC-LAMP $(+)$ mature dendritic cells in combination with activated $\mathrm{T}$ lymphocytes infiltrating primary cutaneous melanoma is a strong independent prognostic factor. Cancer Immunol Immunother. 2007:56:1459-1469.

25. Engelhardt JJ, Boldajipour B, Beemiller P, et al. Marginating dendritic cells of the tumor microenvironment cross-present tumor antigens and stably engage tumor-specific T cells. Cancer Cell. 2012;21:402-417.

\section{Reprint requests:}

Estela Kaminagakura, DDS, PhD

Department of Bioscience and Oral Diagnosis

Science and Technology Institute

University of São Paulo State

Av. Eng. Francisco José Longo

n 777 - Jardim São Dimas

12245-000 - São José dos Campos

SP, Brazil

estela@fosjc.unesp.br 